\title{
Partial Discharge Characterization based on Leakage Current Pulses Waveform for Contaminated Glass Insulator String
}

\author{
N.A. Othman, M.A.M. Piah, Z. Adzis, and Nasir A. Al-geelani \\ Institute of High Voltage \& High Current, Faculty of Electrical Engineering, Universiti Teknologi Malaysia
}

\section{Article Info}

Article history:

Received Oct 23, 2015

Revised Dec 11, 2015

Accepted Jan 4, 2016

\section{Keyword:}

Glass insulator string

Leakage current

Partial discharge

\begin{abstract}
This paper deals with the analysis of partial discharge (PD) occurrences based on leakage current (LC) pulses distribution under different level of contamination. In this work, laboratory based contamination performance tests are conducted on four units of glass insulator string stacked in Iposition. Results show that PD can be characterized (according to their occurrence with respect to the AC supply) as follows: corona discharge occurring dominantly in lightly contaminated insulators, surface discharge occurring dominantly in medium contaminated insulators and finally internal discharge occurring dominantly in heavily contaminated insulators. The ability to distinguish the type of PDs from the LC waveform could be used to monitor and predict the condition of the insulator. The outcome can be incorporated in a conditioning monitoring system.
\end{abstract}

Copyright $\odot 2016$ Institute of Advanced Engineering and Science. All rights reserved.

\section{Corresponding Author:}

N.A. Othman,

Institute of High Voltage \& High Current,

Faculty of Electrical Engineering,

Universiti Teknologi Malaysia

Email: nazlin7@live.utm.my

\section{INTRODUCTION}

Insulator strings that widely used for suspension overhead transmission and distribution lines have provided excellent services for decades. The primary function of overhead insulators is to mechanically support high voltage (HV) cables and electrically isolating them from the grounded structure. However, overhead insulators are often beset crucial problems when dealing with contamination [1]. The voltage distribution of dry and contaminated insulators has uniform resistivity in the absence of water [2]; but in wet condition, uneven resistance exists. The wet surface of contaminated insulator due to high humidity, light rain and fog may lead to the formation of conductive layer and cause leakage current (LC) to flow.

It is an accepted fact that the discharge on the surface of wetly contaminated insulators is directly related to the dry bands [3]. The uneven deposition of contamination sources causes some parts of the insulator to have highest current density especially around the insulator pin [4]. These regions dry more quickly and their resistance increases due to a large amount of heat generated, thus leading to the formation of dry bands. The dry bands existence may lead to the propagation of arcs on the insulator surface which at a certain period of time this arcs may elongate until they bridge the electrodes and finally direct to total flashover.

Dry band arcing plays a negative role in the insulation performance. The appearance of dry bands as a result of Joule effect due to the flow of LC on the contamination layer located on the insulator surface may modify the voltage distribution along the insulator surface. Most of the applied voltage will be distributed at this region since it has high resistance. Localized arcing will be developed if this dry band region cannot withstand the voltage [3]. Further, the formation of dry band distorts the electric field within the band under influence of any moisture and leads to occurrence of partial discharge (PD). 
As quoted from IEC 60270, PD is defined as localized electrical discharges that only partially bridges the insulation between conductors and which can or cannot occur adjacent to a conductor [5]. In accordance with the standard, PD pulse is current or voltage pulse that results from PD occurring within the object under test. Therefore, the LC pulses can be potentially used to indicate the occurrence of PD, referring to the concept applied in PD measurement itself. Although PD does not cause immediate breakdown of the insulation, the appearance of PD indicates the presence of default which cause energy dissipation and further leads to insulation degradation [6,7]. It is therefore, important to diagnose the existence of PD activities so that early prevention action can be taken into consideration.

Literature has shown that LC is the most efficient technique to monitor and investigate the condition of insulator surface. Numerous methods have been used to measure the LC flows on the service insulators; a current transformer (CT) was used in [8] to measure the LC flow on five units of string insulators under contamination test; a combination of CT and shunt resistor was used by [9] to study the flow of LC on the insulators of $400 \mathrm{kV}$ transmission lines. Researchers [10] utilized the advantages of optical fiber as it is immune to electromagnetic interference for their online LC measurement at $500 \mathrm{kV}$ field tower as well as $230 \mathrm{kV}$ and $500 \mathrm{kV}$ substation towers. The surface current of transmission line insulators was observed in [11] using antenna. A novel broadband microcurrent transducer was applied in online measurement of LC system by [12]. The flow of LC of the insulators available on $275 \mathrm{kV}$ line in YTL Power Station was investigated by [13] using artificial electrode. Various methods were implemented as described above to investigate the flow of LC. This shows that the presence of LC is crucial and may lead to arcing activities on the surface of insulators, which may result to contamination flashover.

Therefore, an attempt to characterize the type of PD based on LC pulses waveform is presented in this paper. A string of four glass insulators (without grading ring) used as main sample was contaminated with different levels of contamination. The flow of LC is measured by using a 100 shunt resistor at the grounded end of the string insulators and analyzed by superimposed compilation of 10 cycle current pulse events.

\section{RESEARCH METHOD}

Salt particularly ammonium chloride with the weights of $10 \mathrm{~g}, 50 \mathrm{~g}$, and $100 \mathrm{~g}$ were dissolved in 1 liter of distilled water to form different saline solutions and subsequently tested for contamination level using Equivalent Salt Deposit Density (ESDD) method. Four units of HV glass insulators were pre-contaminated with those different saline solutions and were completely dried for 24 hours before entering the test chamber for voltage injection. Full measurement setup which is modified from [14] is illustrated in

Figure 1 (a) with the insulators suspended vertically while the pictorial view of the experimental setup is depicted in $60507[15]$

Figure 1 (b). The laboratory experiment for artificial pollution tests was conducted based on IEC

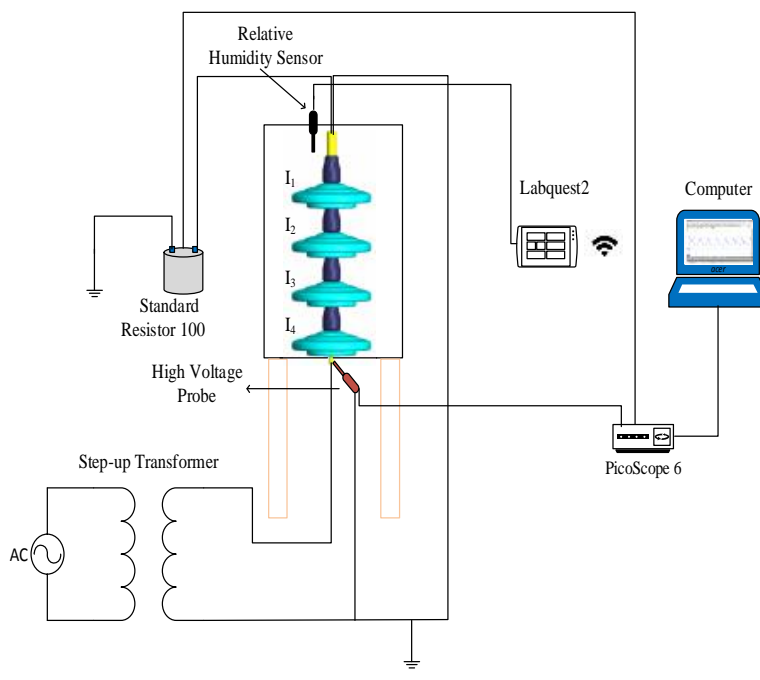

(a)

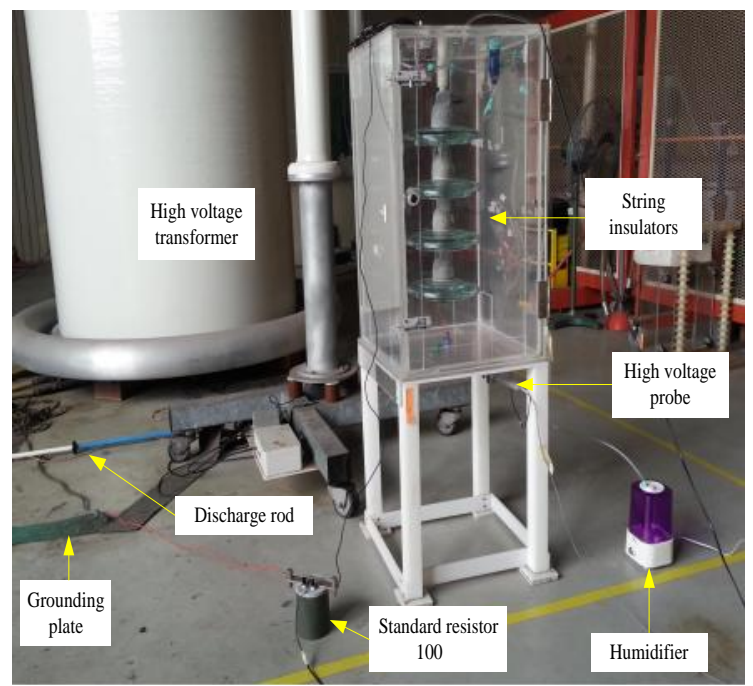

(b) 
Figure 1. (a) Full measurement setup and (b) pictorial view of experimental setup

The operating voltage of $33 \mathrm{kV}$ AC was applied when the contamination layer is completely wetted by clean steam fog as depicted in Figure 2 and the relative humidity in the range of $80 \%$ to $100 \%$. This humidity range is chosen by taking into account the fact that the surface conductivity often increases when the relative humidity exceeds $75 \%$ [16]. The flow of LC was measured by a 100 standard resistor at the grounded end of the string insulators without any electrical contact with the HV supply. The measured LC is then continuously captured by PicoScope ${ }^{\circledR}$ and subsequently recorded by a computer, along with the HV probe measured AC supply.

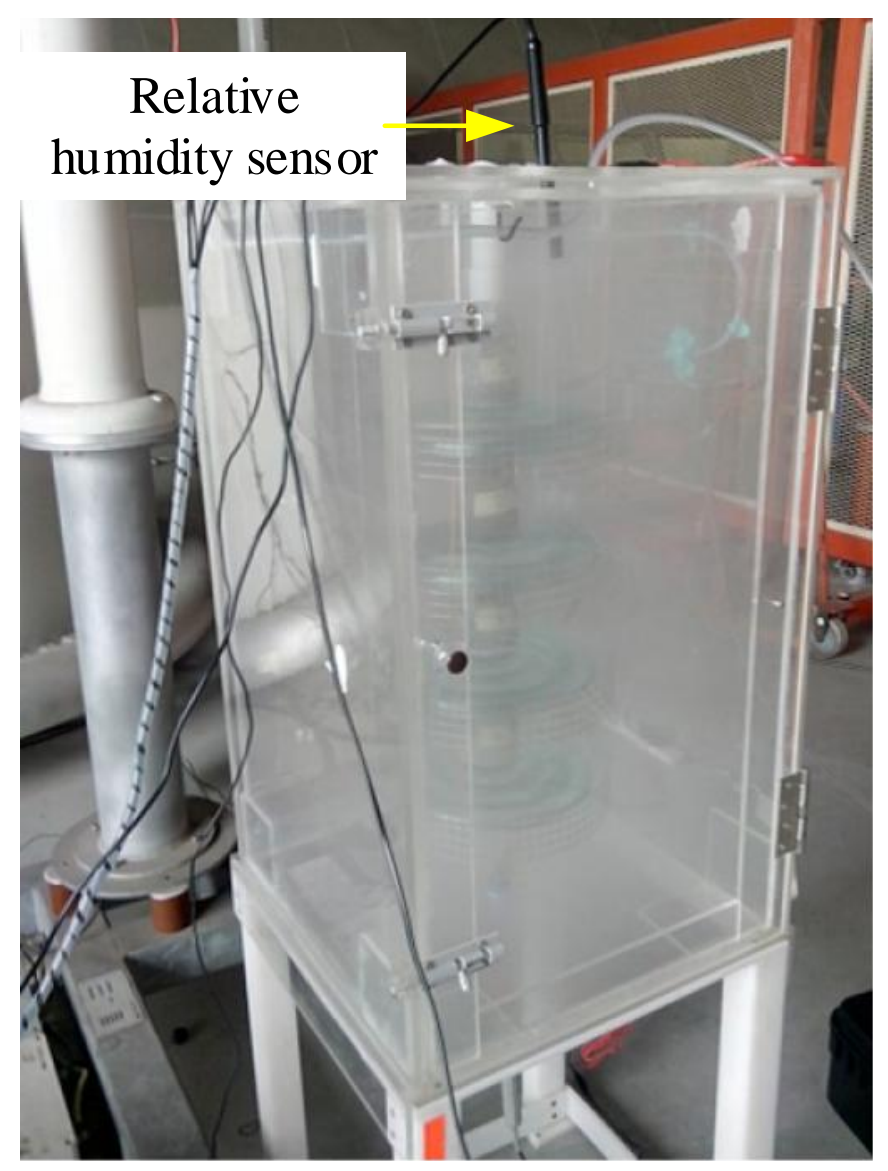

Figure 2. Insulators wetted by clean steam fog

\section{RESULTS AND ANALYSIS}

Table 1 presents the contamination level of the tested saline solution according to an application guide for contaminated insulators proposed by CIGRE [17]. Based on this result, different levels of contamination are obtained and the insulator was subsequently contaminated with ammonium chloride.

Table 1. Contamination level according to saline solution

\begin{tabular}{ccc}
\hline Salt weight $(\mathrm{g})$ & ESDD $\left(\mathrm{mg} / \mathrm{cm}^{2}\right)$ & Contamination level [17] \\
\hline 0 & 0.00 & Clean \\
10 & 0.05 & Light \\
50 & 0.07 & Medium \\
100 & 0.13 & Heavy \\
\hline
\end{tabular}

\subsection{Clean String Insulator}


Error! Reference source not found. shows the leakage current (LC) waveform for clean insulator string. From the figure, there is a small pulse on the negative cycle of applied voltage. Small pulse of LC which is less than $1 \mathrm{~mA}$ peak can be considered as typical noise [18]. This finding suggests that in clean and dry conditions, no LC flows along the insulator surface.

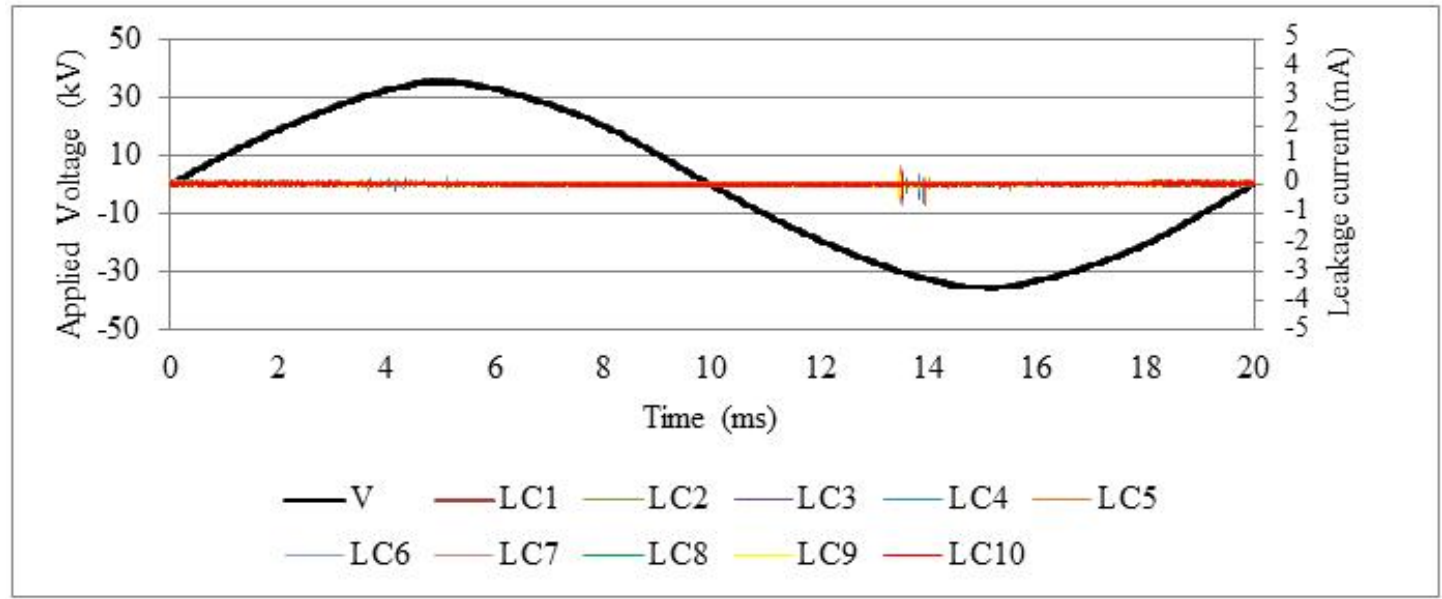

Figure 3. Leakage current pulses waveform for clean insulator string

\subsection{Lightly Contaminated String Insulator}

Error! Reference source not found. presents the LC pulses waveform for lightly contaminated insulator string. It is apparent that the LC pulses occur at the phase angle of 90 degrees in both cycles of applied voltage. The presented result can be characterized as corona discharge, as the distribution of LC pulses occurs in the range of 80 to 100 degree phase of applied voltage [19]. The appearance of higher LC pulses in the negative cycle of applied voltage is due to the formation of negative corona discharge and supported by [20]. This situation is due to the mobility of free electron is much faster than ion under the influence of applied voltage. Less LC activities occurred at the positive cycle may be explained by considering that the inception voltage for positive corona is higher compared to negative corona [21]. It can thus be suggested that both (positive and negative) corona discharge occurs when the string insulators were lightly contaminated and agreed by [22]. Also can be concluded that the LC pulses pattern for corona discharge is not symmetrical in which negative corona has lower inception voltage than positive corona. It is also noticed from the presented result that LC pulses appear around the applied voltage spike with the range of amplitude between $0.02 \mathrm{~A}$ to $0.05 \mathrm{~A}$.

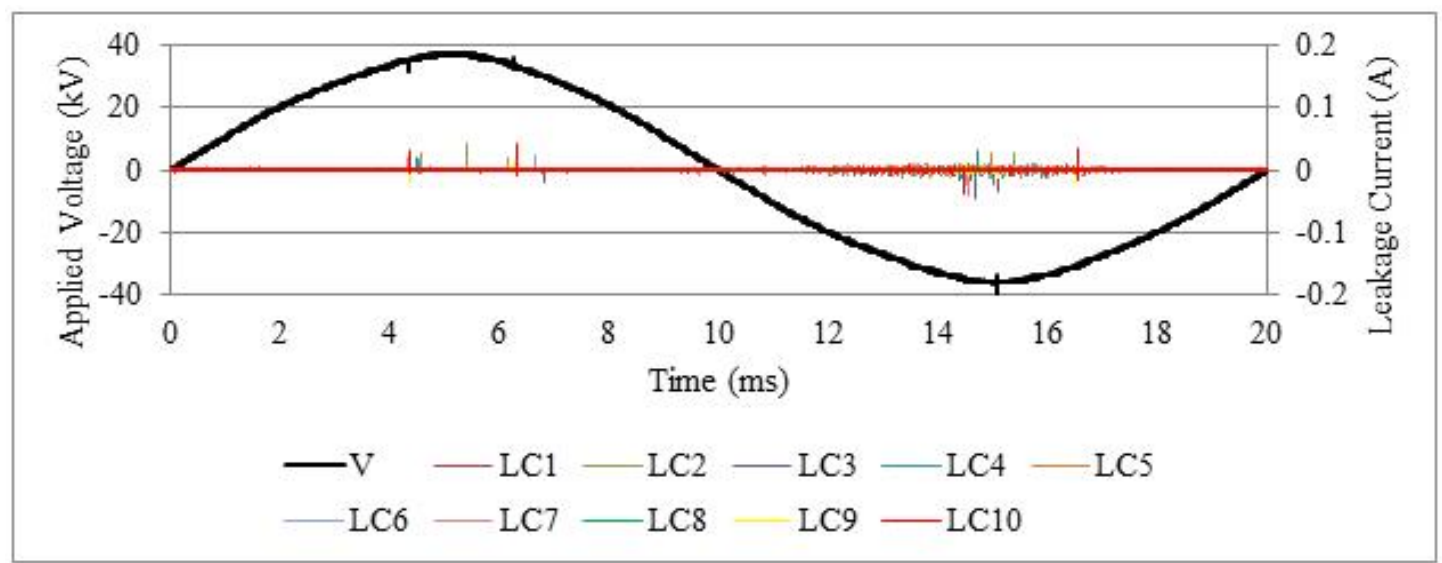

Figure 4. Leakage current pulses waveform for lightly contaminated insulator string 


\subsection{Medium Contaminated String Insulator}

Figure 5 illustrates the LC pulses waveform for medium contaminated insulator string. It is clear that the LC pulses are obtained at the location from 0 to 90 degree phase for positive cycle of applied voltage while 180 to 270 degree phase for negative cycle of applied voltage. The presented result corroborates the findings of researcher [23, 24] who classify discharge pattern distributed within the first and third phase quadrant of applied voltage as surface discharge. Surface discharge may occur due to the presence of high tangential field. The non-uniform contamination layer along the surface of glass insulator applied during the experiment could lead to the formation of this tangential field and enable the occurrence of surface discharge. It can therefore be assumed that the surface discharge may occur in the glass string insulator during medium contaminated conditions. It is also can be concluded that the LC pulses pattern for surface discharge in this work is symmetrical between positive and negative cycles. It is worth to mention that the LC appears around the applied voltage spike with the range of amplitude is between 0.06A to $0.12 \mathrm{~A}$.

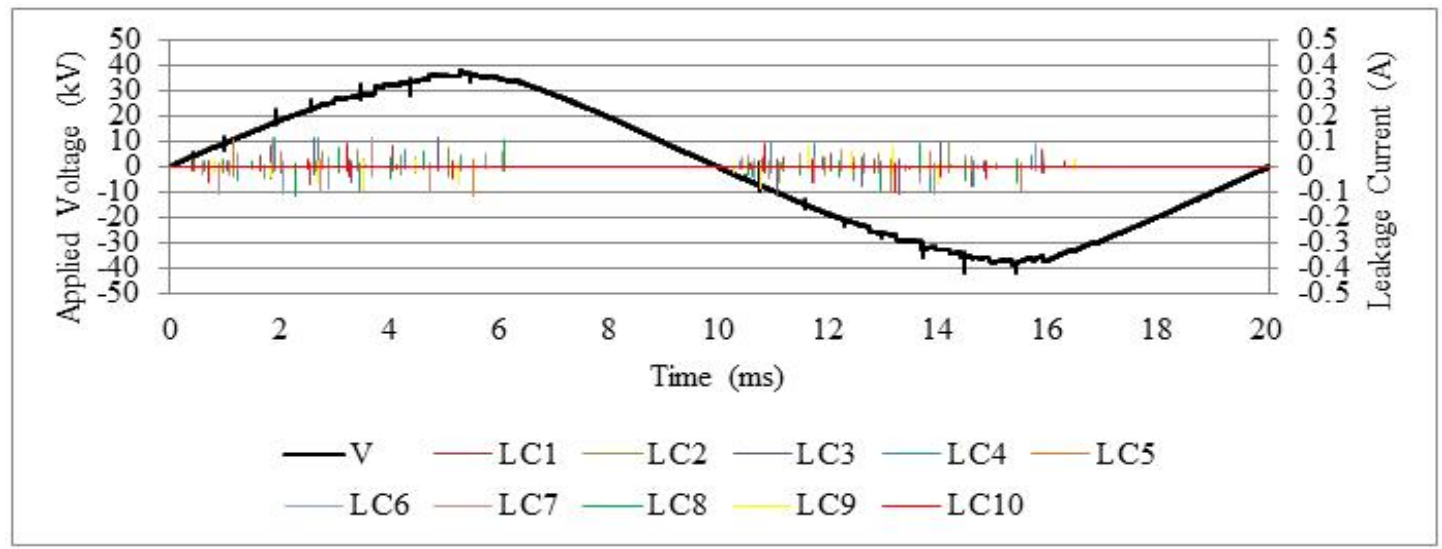

Figure 5. Leakage current pulses waveform for medium contaminated insulator string

\subsection{Heavily Contaminated String Insulator}

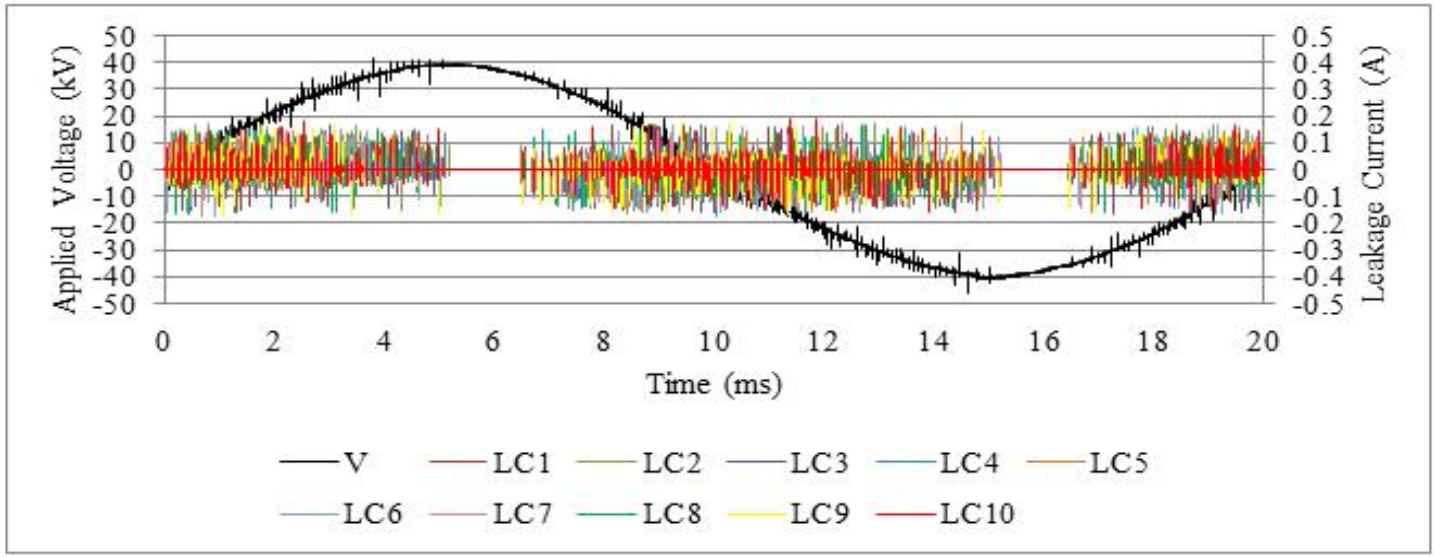

Figure 6 depicts the LC pulses waveform for heavily contaminated insulator string. The LC pulses appear again at the location of applied voltage spike with the range of amplitude between $0.16 \mathrm{~A}$ to $0.19 \mathrm{~A}$. Interestingly, the LC pulse occurs at the phase of $0^{\circ}$ to $90^{\circ}$ and $135^{\circ}$ to $180^{\circ}$ for positive cycle of applied 
voltage while $180^{\circ}$ to $270^{\circ}$ and $315^{\circ}$ to $360^{\circ}$ for negative cycle of applied voltage. Referring to

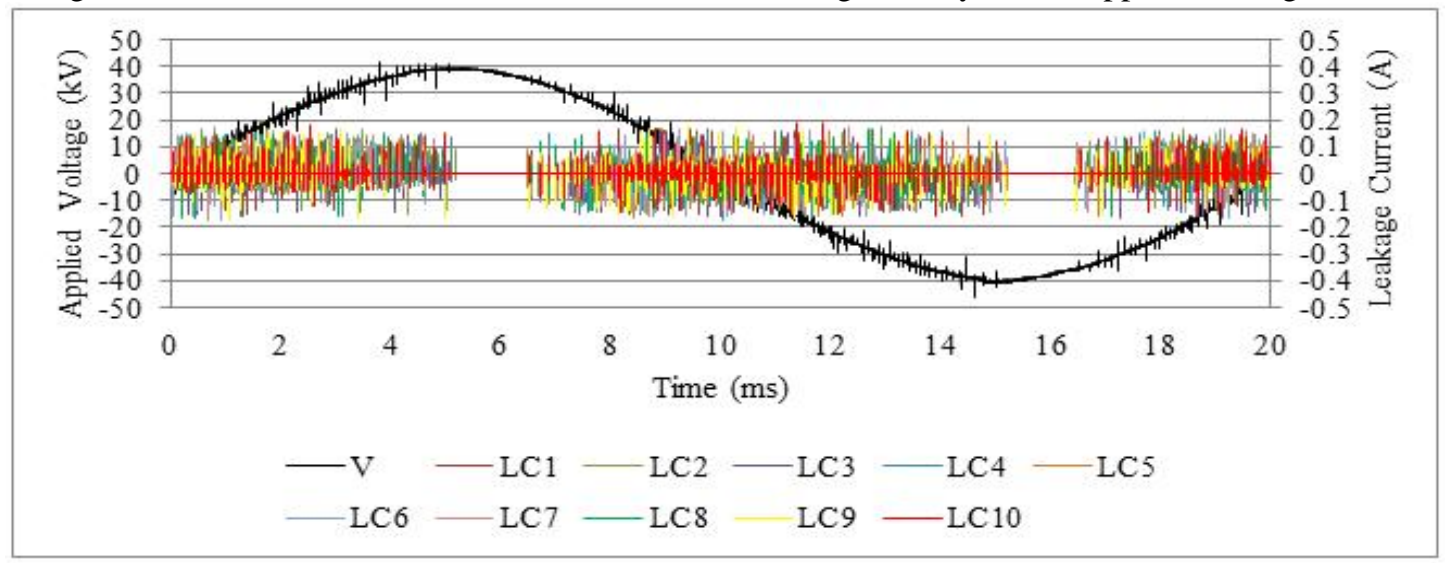

Figure 6, the distribution of LC pulses can be characterized as internal discharge as it has similar distribution of PD charge magnitude [20]. The result also appears to be consistent with the simulation study conducted by [25] in the void modeling using Monte-Carlo simulation. Their finding shows that the charge is distributed on the insulation surface initially, and then followed by a uniform distribution in the void and finally internal discharge occurs.

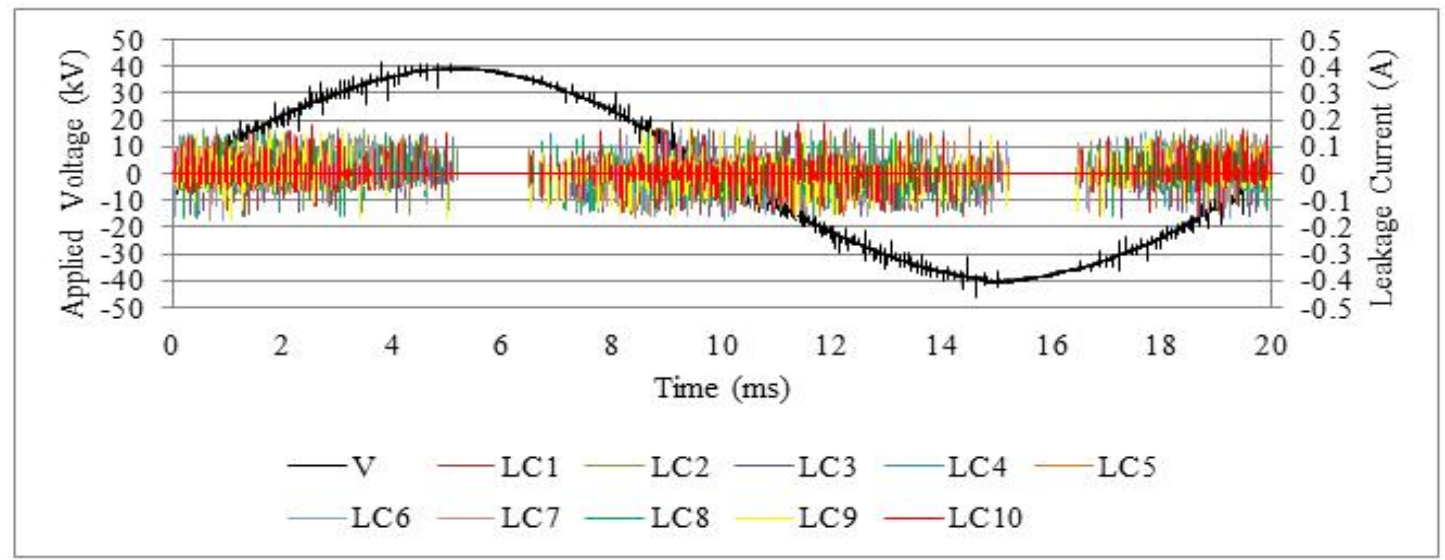

Figure 6. Leakage current pulses waveform for heavily contaminated insulator string

It is well understood that internal discharge usually occurs in the void that has lower breakdown strength than solid insulation [26]. However, in this work, it is important to emphasize that internal discharges are initiated due to high electric concentration field between contamination layer and the glass insulator surface. The occurrence of dry band region on the glass insulator surface could be another possible explanation for internal discharge event during heavily contamination. The internal PD can be initiated when there is a small glow discharge burning in the dry band region [16]. In this work therefore, there may be glow discharge which cannot be seen directly by eye but detected by LC measurement. It is also important to mention that the existence of LC flow causes glow and heat that can be detected by other methods such as infrared (IR) or thermal camera.

The presented results have contributed to the knowledge of the type's dominant PD occurrences based on LC pulses distribution according to the contamination level with respect to the applied voltage waveform. This knowledge can be used by service engineer to monitor the condition of string insulators while in service and time to replace the insulators can be predicted. The dominant types of PD based on LC pulses distribution with respect to applied voltage waveform according to the contamination level is summarized in Table 2.

Table 2. The distribution of LC pulses with respect to applied voltage waveform according to the contamination level

\begin{tabular}{lcc}
\hline Contamination Level & Phase Angle & Types of Partial \\
\hline
\end{tabular}




\begin{tabular}{cccc}
\hline & Positive cycle & Negative cycle & Discharge (dominant) \\
\hline Clean & - & - & No discharge \\
Light & $80^{\circ}$ to $100^{\circ}$ & $260^{\circ}$ to $280^{\circ}$ & Corona discharge \\
Medium & $0^{\circ}$ and $90^{\circ}$ & $180^{\circ}$ to $270^{\circ}$ & Surface discharge \\
Heavy & $0^{\circ}$ to $90^{\circ}$ and $135^{\circ}$ to $180^{\circ}$ & $180^{\circ}$ to $270^{\circ}$ and $315^{\circ}$ to $360^{\circ}$ & Internal discharge \\
\hline
\end{tabular}

\subsection{Leakage Current Amplitude}

Based on the findings obtained from Section 3.1 to 3.4, it is perceived that the LC pulses amplitude increase as the contamination level increases. Therefore, this section will discuss on the maximum value of LC flowing through the string insulator under all conditions. Maximum value of LC flows along the surface of insulator string with its phase angle under different levels of contamination is shown in Figure 7. It is apparent form this figure that the value of LC magnitude increases as the contamination level is increased. This observation is true since the LC pattern extracted from [27] also increases when the insulator becomes more contaminated. It is also worth to mention that the value of $\mathrm{LC}$ is in root-mean-square value.

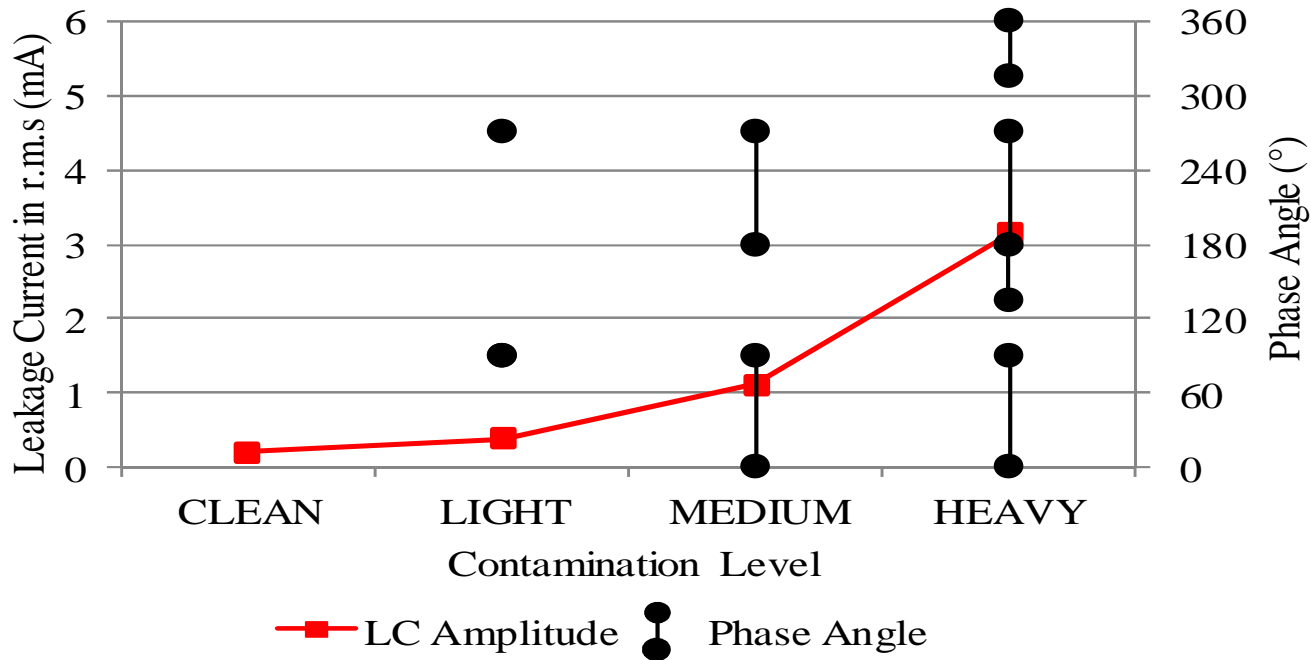

Figure 7. Maximum value of LC amplitude with phase angle of applied voltage waveform

Another interesting observation can be seen from Figure 7 is that the LC pulses distribution according to the phase angle of applied voltage during heavily contaminated condition is overlapped with lightly and medium contaminated conditions. This shows that, internal discharge occurs along with corona and surface during heavily contaminated conditions but is dominated by the internal discharge. Similar situation also occurs during medium contaminated conditions where the distribution of LC pulses for corona and surface discharge overlapped at 90 degree of applied voltage. This situation indicates that the occurrence of surface discharge during medium contaminated comes with corona but surface discharge event is more dominant.

\section{CONCLUSION}

The present article provides the characterization of partial discharge based on leakage current pulses waveform. Based on the presented results, PD can be characterized as follows: corona discharge occurs dominantly in lightly contaminated insulators, surface discharge is dominant in medium contaminated insulators and finally internal discharge occurs dominantly in heavily contaminated insulators. The ability to distinguish the type of discharge from the LC waveform could be used to monitor and predict the condition of the insulator while energized and in service. Therefore, an immediate action can be taken thus increasing the reliability of the power system network. It is expected that when the LC pulses are distributed at phase degrees of $0^{\circ}$ to $360^{\circ}$ (all four quadrant), it can be used as a pre-warning of an impending breakdown. 


\section{ACKNOWLEDGEMENTS}

The authors would like to thank Universiti Teknologi Malaysia (UTM), for the use of facilities and by awarding a research grants under vote 01H69, 03H86, 4L133 and 4F751 from the Research Management Centre UTM, and Malaysia Ministry of Higher Education (MOHE).

\section{REFERENCES}

[1] R. Wilkins. Flashover voltage of high-voltage insulators with uniform surface-pollution films. Proceedings of the Institution of Electrical Engineer. 1969; 116: 457-465.

[2] Othman N.A, Piah M.A.M, Adzis Z, Ahmad H, and Ahmad N. Simulation of voltage and electric-field distribution for contaminated glass insulator. IEEE Student Conference on Research and Development (SCOReD). Putrajaya. 2013: 116-120.

[3] Zhou J.B, Gao B, and Zhang Q.G. Dry band formation and its influence on electric field distribution along polluted insulator. Asia-Pacific Power and Energy Engineering Conference (APPEEC). 2010: 1-5.

[4] Williams D, Haddad A, Rowlands A, Young H, and Waters R. Formation and characterization of dry bands in clean fog on polluted insulators. IEEE Transactions on Dielectrics and Electrical Insulation. 1999;6: 724-731.

[5] B.S. Institution. High-voltage test techniques. Partial discharge measurements. in BS EN 60270, ed. London, 2001.

[6] Zhu M, Chen G, Xu M, Liu T, Xie D, and Zhang Y. Study on monitoring system for partial discharge of electrical equipment. TELKOMNIKA Indonesian Journal of Electrical Engineering. 2014; 12: 1053-1059.

[7] Weixia Z, Xianping Z, K, Shutao Z, Hong Y, and Dada W. Study on partial discharge detection of 10kV XLPE power cable. TELKOMNIKA Indonesian Journal of Electrical Engineering. 2012; 10: 1795-1799.

[8] Suda T. Frequency characteristics of leakage current waveforms of a string of suspension insulators. IEEE Transactions on Power Delivery. 2005; 20: 481-487.

[9] Fierro-Chavez J.L, Ramirez-Vazquez I, and Montoya-Tena G. On-line leakage current monitoring of $400 \mathrm{kV}$ insulator strings in polluted areas. IEE Proceedings-Generation, Transmission and Distribution. 1996; 143: 560564.

[10] Oliveira S.C, Fontana E, and Monte de Melo Cavalcanti F.J. Leakage current activity on glass-type insulators of overhead transmission lines in the northeast region of Brazil. IEEE Transactions on Power Delivery. 2009; 24 : 822827.

[11] Youssef M. and El-Alayly A.A. Remote monitoring of surface current over insulators. IEEE/PES Transmission and Distribution Conference and Exposition. 2001; $1: 113-116$.

[12] Weigen C, Chenguo Y, Pan C, Caixin S, Lin D, and Ruijin L. A new broadband microcurrent transducer for insulator leakage current monitoring system. IEEE Transactions on Power Delivery. 2008; 23: 355-360.

[13] Salam M.A, El-Fadil N, and Nadir Z. A novel approach to predict leakage current in insulators from wind velocity. ECTI Transaction on Electrical Eng., Electronics and Communications. 2006; 4: 47-50.

[14] Othman N.A, Piah M.A.M, Adzis Z, Ahmad H, Ahmad N.A, Kamarden H, and Suleiman A.A. Characterization of charge distribution on the high voltage glass insulator string. Journal of Electrostatics. 2014; 72: 315-321.

[15] B.S. Institution. Artificial pollution tests on high-voltage ceramic and glass insulators to be used on a.c. systems. in BS EN 60507, ed. London, 2014.

[16] Chrzan K.L. and Moro F. Concentrated discharges and dry bands on polluted outdoor insulators. IEEE Transactions on Power Delivery. 2007; 22: 466-471.

[17] A Critical Comparison Of Artificial Pollution Test Methods For HV Insulators. CIGRE Taskforce 33-04. 1979; 64: 117-136.

[18] Pylarinos D, Siderakis K, Pyrgioti E, Thalassinakis E, and Vitellas I. Investigation of Leakage Current Waveforms Recorded in a Coastal High Voltage Substation. Engineering, Technology \& Applied Science Research (ETASR). 2011; 1: 63-69.

[19] Nattrass D.A. Partial discharge measurement and interpretation. IEEE Electrical Insulation Magazine. 1988; 4: 1023.

[20] Illias H, Teo Soon Y, Bakar A.H.A, Mokhlis H, Chen G, and Lewin P.L. Partial discharge patterns in high voltage insulation. IEEE International Conference on Power and Energy (PECon). 2012: 750-755.

[21] F.H. Kreuger, Industrial High DC Voltage: Delft University Press, 1995.

[22] Bologna F.F, Reynders J.P, and Britten A.C. Corona discharge activity on a string of glass cap-and-pin insulators under conditions of light wetting, light non-uniform contamination. IEEE Bologna Power Tech Conference Proceedings. 2003; $3: 8$.

[23] Kreuger F.H, Gulski E, and Krivda A. Classification of partial discharges. IEEE Transactions on Electrical Insulation. 1993; 28: 917-931.

[24] Wanting D, Zhong Z, Ling R, Yu S, Qijia X, and Hui W. Power apparatus insulation diagnosis through partial discharge in a smarter grid. Conference Record of the 2010 IEEE International Symposium on Electrical Insulation (ISEI). 2010: 1-4

[25] Wu K, Suzuoki Y, and Dissado L. A. Improved simulation model for PD pattern in voids considering effects of discharge area. Conference on Electrical Insulation and Dielectric Phenomena. 2003: 32-35.

[26] M.S. Naidu and V. Kamaraju, High Voltage Engineering, Fifth Edition ed. New Delhi: McGraw Hill Education (India) Private Limited, 2013. 
[27] Chandrasekar S, Kalaivanan C, Cavallini A, and Montanari G.C. Investigations on leakage current and phase angle characteristics of porcelain and polymeric insulator under contaminated conditions. IEEE Transactions on Dielectrics and Electrical Insulation. 2009; 16: 574-583.

\section{BIOGRAPHIES OF AUTHORS}
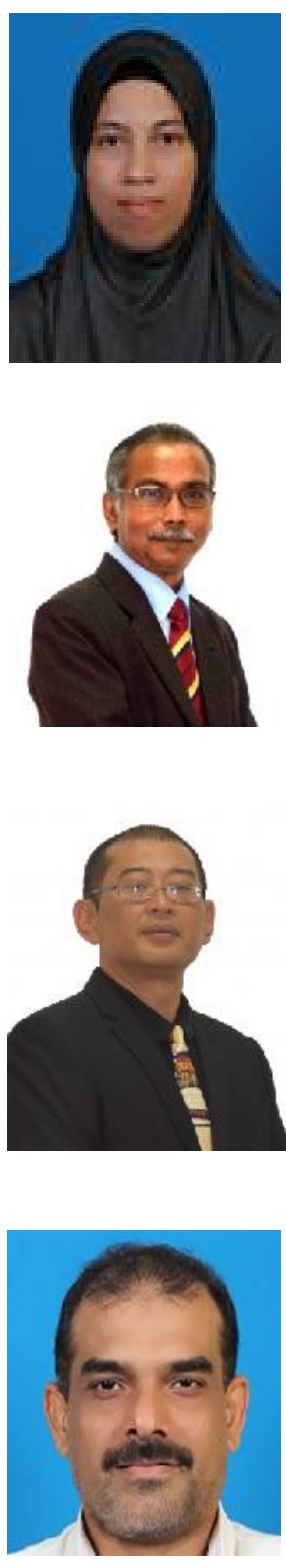

N.A. Othman was born in Johor, Malaysia on January 19, 1986. She received her Electrical Engineering (Communication) diploma degree from Universiti Teknologi Malaysia (UTM), Kuala Lumpur in 2007 and received B.Eng in Electrical Engineering from UTM, Skudai in 2010. She is currently pursuing Ph.D. degree at Institute of High Voltage and High Current (IVAT) in Faculty of Electrical Engineering, UTM. Her research interest includes the detection and diagnostics of partial discharges and space charge in insulation for condition monitoring.

Mohamed Afendi Mohamed Piah was born in Perak, on November 8, 1963. He is an associate professor at Faculty of Electrical Engineering, Universiti Teknologi Malaysia (UTM) and a fellow member of the Institute of High Voltage and High Current (IVAT). He received the B.Elect. Eng. degree from UTM in 1986, M.Sc in Power System from University of Strathclyde, UK in 1990 and PhD in High Voltage Engineering from UTM in 2004. His research interests include high voltage insulation diagnostic and co-ordination, electrical discharges, polymer nanocomposites insulating materials and insulator condition monitoring.

Zuraimy Adzis was born in Singapore, in 1971. He graduated from University of Salford, Manchester in 1995. He received the M.Eng. Degree from the Universiti Teknologi Malaysia, in 2003. Currently, he is a lecturer in Institute of High Voltage and High Current, Universiti Teknologi Malaysia, Johor Bahru, Malaysia. His research interest includes EMC in high voltage Systems, with focus in lightning interaction and partial discharge phenomenon.

Dr. Nasir Ahmed Algeelani received the B.E. degree in electrical power system from University of Aden, Yemen, Aden, in 1997, the M.E. degree in electrical power system engineering from University Technology Malaysia in 2009 and the Ph.D. degree in high voltage engineering from University Technology Malaysia in 2014. He was a Lecturer with Industrial Technical Institute (ITI) for 25 years, where he is currently a senior lecturer of High Voltage Engineering. At the present he is a postdoctoral candidate at high voltage engineering department at University Technology Malaysia. He has published as authored and co-authored more than 30 papers in various technical journals and conference proceedings. His research interests include highvoltage instrumentation, partial discharge, detection and warning systems and condition monitoring of high power equipment. 